\title{
Magnetic-field-induced biaxiality in an antiferroelectric liquid crystal
}

\author{
I. Muševič, B. Žekš, and R. Blinc \\ J. Stefan Institute, Jamova 39, 61000 Ljubljana, Slovenia \\ Th. Rasing \\ Research Institute for Materials and High Field Magnet Laboratory, University of Nijmegen, NL-6525 ED Nijmegen, The Netherlands
}

(Received 23 September 1992)

\begin{abstract}
The magnetic-field-induced biaxiality of the originally uniaxial antiferroelectric chiral smectic (Sm$C_{A}^{*}$ ) phase of 4-(1-methyl-heptyloxycarbonylphenyl) 4'-octylbiphenyl-4-carboxylate (MHPOBC) has been studied in a magnetic field applied perpendicular to the helical axis. The observed distortions of the central part of the conoscopic figures are explained on the basis of a perturbative approach to light propagation in spatially modulated birefringent phases. The critical magnetic field for the unwinding of the helical structure of the $\mathrm{Sm}-C_{A}^{*}$ phase of $\mathrm{MHPOBC}$ is $13 \mathrm{~T}$ at $89^{\circ} \mathrm{C}$ and is strongly temperature dependent.
\end{abstract}

PACS number(s): 61.30.Gd, 42.25.Bs, 64.70.Md, 64.70.Rh

\section{INTRODUCTION}

In the antiferroelectric Sm- $C_{A}^{*}$ phase of a chiral smectic liquid crystal, molecules in two adjacent layers are tilted with respect to the layer normal $\mathbf{e}_{z}$ at a tilt angle $\pm \theta$. The electric dipole moments $\mathbf{P}$ of molecules in the two neighboring smectic layers are on the average nearly but not exactly antiparallel. They form an angle $\psi=\pi+\delta$ $(\delta<1)$, resulting in a very small value of the local dipole moment. Due to the chirality of liquid-crystalline molecules, the molecular directors in the $\mathrm{Sm}-C_{A}^{*}$ precess around $\mathbf{e}_{z}$ as one moves along the layer normal. As a result, one can consider the $\mathrm{Sm}-C_{A}^{*}$ structure a doublehelical structure, formed of two identical, slightly displaced helices gearing into each other along the $z$ axis. The length of the period $p$ of the double helical structure is of the order of the wavelength of visible light and is much larger than the interlayer distance, so that the undisturbed Sm- $C_{A}^{*}$ phase represents a helicoidal continuum with the optical axis parallel to the direction of the helix. The symmetry element of each smectic layer is $C_{2}$.

In the last few years the antiferroelectric Sm- $C_{A}^{*}$ phase and the related phases $\mathrm{Sm}-C_{\alpha}^{*}$ and $\mathrm{Sm}-C_{\gamma}^{*}$ have been the subject of intensive research, inspired by the very interesting thermodynamical, optical, and electrooptical properties of these phases. In particular, optical conoscopy has been applied [1-3] to study the response of a thick, homeotropically aligned $\mathrm{Sm}-C_{A}^{*}$ sample to an external electric field. An in-plane electric field was shown to distort the conoscopic figure, resulting in a characteristic biaxial figure at very high fields. The axis of the smallest index of refraction of the induced biaxial structure was found to lie in the plane, perpendicular to the field direction, whereas the conoscopic figure was centered around the layer normal $\mathbf{e}_{z}$. These observations suggested that the dielectric coupling was responsible for the observed distortion. In addition, it has been observed that the conoscopic figure was not saturated even at electric-field strengths as high as $20 \mathrm{kV} / \mathrm{cm}$, which is one to two orders of magnitude larger than the critical electric field for the unwinding of the helix in the ferroelectric $\mathrm{Sm}-C^{*}$ phase of the same material.

The motivation for the present work is the lack of a complete theoretical description of the distortion of the conoscopic figure of the Sm- $C_{A}^{*}$ in an external field. In view of the extremely high electric fields, necessary to unwind the antiferroelectric phase, as well as the presence of the dielectric and ferroelectric couplings in an external electric field, we have carried out an experiment on the antiferroelectric $\mathrm{Sm}-C_{A}^{*}$ phase of 4-(1-methylheptyloxycarbonylphenyl) 4'-octylbiphenyl-4-carboxylate (MHPOBC) in an external magnetic field, applied perpendicular to the helix. The observed distortion of the conoscopic figure of MHPOBC is typical for a material with positive diamagnetic anisotropy and can be well described by a simple perturbative approach.

\section{THEORY}

The optical properties of the Sm- $C_{A}^{*}$ phase are characterized by the dielectric tensor $\epsilon_{A}(\mathbf{r})$ of the double-helical structure, which is formed by two identical, slightly displaced ferroelectric Sm- $C^{*}$ helices. The local value of the dielectric tensor $\epsilon_{A}(\mathbf{r})$ is obtained [4,5] by two successive rotations of the tensor with the principal axis $\epsilon_{i}$, $i=1,2,3$, starting from the configuration, where the eigenframe $(1,2,3)$ coincides with the laboratory frame $(x, y, z)$. First the tensor is rotated through the tilt angle $\theta$ around the $y$ axis followed by a rotation through the phase angle $\Phi(z)$ around the $z$ axis.

Keeping in mind that the interlayer distance of the Sm- $C_{A}^{*}$ phase is several orders of magnitude smaller than the wavelength of the light of interest, we may assume that the dielectric tensor $\epsilon_{A}$ of the Sm- $C_{A}^{*}$ phase can be well approximated by the dielectric tensor $\underline{\epsilon}(z)$, which is given by the average value of the Sm- $C_{A}^{*}$ dielectric tensor in two neighboring layers. In the limit of small $\theta$, this $\underline{\epsilon}(z)$ can be written as 


$$
\begin{aligned}
& \underline{\epsilon}(z)=\underline{\epsilon}_{0}+\delta \underline{\epsilon}=\left[\begin{array}{ccc}
\frac{1}{2}\left(\epsilon_{1}+\epsilon_{2}\right)+\frac{1}{2}\left(\epsilon_{3}-\epsilon_{1}\right) \theta^{2} & 0 & 0 \\
0 & \frac{1}{2}\left(\epsilon_{1}+\epsilon_{2}\right)+\frac{1}{2}\left(\epsilon_{3}-\epsilon_{1}\right) \theta^{2} & 0 \\
0 & 0 & \epsilon_{3}-\left(\epsilon_{3}-\epsilon_{1}\right) \theta^{2}
\end{array}\right] \\
& +\frac{1}{2}\left[\left(\epsilon_{1}-\epsilon_{2}\right)+\left(\epsilon_{3}-\epsilon_{1}\right) \theta^{2}\right]\left[\begin{array}{ccc}
\cos 2 \Phi(z) & \sin 2 \Phi(z) & 0 \\
\sin 2 \Phi(z) & -\cos 2 \Phi(z) & 0 \\
0 & 0 & 0
\end{array}\right] \\
& +\frac{1}{2}\left[\left(\epsilon_{1}-\epsilon_{2}\right)+\left(\epsilon_{3}-\epsilon_{1}\right) \theta^{2}\right] \delta\left[\begin{array}{ccc}
-\sin 2 \Phi(z) & \cos 2 \Phi(z) & 0 \\
\cos 2 \Phi(z) & \sin 2 \Phi(z) & 0 \\
0 & 0 & 0
\end{array}\right] \\
& +\frac{1}{2}\left(\epsilon_{3}-\epsilon_{1}\right) \theta \delta\left[\begin{array}{ccc}
0 & 0 & \sin \Phi(z) \\
0 & 0 & -\cos \Phi(z) \\
\sin \Phi(z) & -\cos \Phi(z) & 0
\end{array}\right]
\end{aligned}
$$

where $\underline{\epsilon}_{0}$ and $\delta \underline{\epsilon}(z)$ are spatially homogeneous and inhomogeneous parts of $\epsilon(z)$, respectively. Here $\Phi(z)$ is the phase of the order parameter and is defined as the angle between the $x$ axis and the projection of the director on the $x-y$ plane, whereas $\delta$ describes the difference $\Delta \Phi$ between the phases of the order parameter in the two neighboring layers, $\Delta \Phi=\Phi(n+1)-\Phi(n)=\pi+\delta$. It is interesting to note that the last two terms in Eq. (1) are proportional to $\delta$, which is expected to be very small in the Sm- $C_{A}^{*}$ phase. As a result, part of the quadrupolar terms $\cos 2 \Phi(z)$ and $\sin 2 \Phi(z)$, and especially the dipolar terms $\cos \Phi(z)$ and $\sin \Phi(x)$ of the dielectric tensor, average to a very small value in the Sm- $C_{A}^{*}$ phase and will be neglected. This has important implications for the optical properties of the antiferroelectric Sm- $C_{A}^{*}$ phase. The first two dominant terms in Eq. (1), which are similar to the dielectric tensor of the chiral nematic phase [6], result in a reflection spectrum [1], characteristic for chiral nematic phases. The only difference is in the magnitude of the inhomogeneous term of the Sm- $C_{A}^{*}$ phase. In view of small intrinsic biaxiality $\epsilon_{1} \approx \epsilon_{2}$, the magnitude of this term is smaller by a factor of the square of the tilt angle $\theta^{2}$ than the corresponding term for chiral nematics.

The spatially inhomogeneous term in the dielectric tensor of the Sm-C $C_{A}^{*}$ phase [Eq. (1)] is typically $10^{-1}$ to $10^{-2}$ smaller than the principal values $\epsilon_{i}$. This suggests that for the optical response of the $\mathrm{Sm}-C_{A}^{*}$ phase, a simple perturbative approach might be used, starting from the exact solutions of the unperturbed homogeneous wave equation $(\delta \underline{\epsilon}=0)$, which are linearly polarized ordinary $(\sigma)$ and extraordinary $(\pi)$ waves. As has been shown by Oldano [7,8], optical properties of tilted smectic phases can indeed be well described by the unperturbed $\sigma$ and $\pi$ linearly polarized eigenmodes of a uniaxial crystal with the corresponding linear dispersion relations $\omega(\mathbf{k}, p)$. Such an approximation breaks down in the case of a degeneration of $\sigma$ and $\pi$ modes. These special degeneration points in $(\mathbf{k}, \omega)$ space include the propagation of light along the helical axis, the propagation of light at the Bragg angle, and the case of the critical value [7,9] of the tilt angle of the smectic phase. We shall limit the discus- sion to the case of nondegenerate $\sigma$ and $\pi$ modes, whereas the degeneration points are beyond the scope of this analysis.

Following the approach of Peterson [10], we define a tensor-weighted inner product of the two eigenfunctions $|\mathbf{k}, p\rangle$ and $\left|\mathbf{k}^{\prime}, p^{\prime}\right\rangle$, characterized by the wave vector $\mathbf{k}$ and polarization $p$ as

$$
\left\langle\mathbf{k}^{\prime}, p^{\prime} \mid \mathbf{k}, p\right\rangle=\left\langle\mathbf{E}_{k^{\prime}, p^{\prime}} \mid \mathbf{E}_{k, p}\right\rangle=\frac{1}{(2 \pi)^{3}} \int d \mathbf{r} \mathbf{E}_{k^{\prime}, p^{\prime}}^{*} \underline{\epsilon}(\mathbf{r}) \mathbf{E}_{k, p} .
$$

Here, $\left|\mathbf{k}^{\prime}, p^{\prime}\right\rangle$ and $|\mathbf{k}, p\rangle$ represent eigensolutions of the wave equation in the inhomogeneous medium, characterized by the dielectric tensor $\underline{\epsilon}(\mathbf{r})=\underline{\epsilon}_{0}+\delta \underline{\epsilon}(\mathbf{r})$,

$$
\underline{\epsilon}_{0}^{-1} \nabla \times \nabla \times|\mathbf{k}, p\rangle-\omega^{2} \underline{\epsilon}_{0}^{-1} \delta \underline{\epsilon}|\mathbf{k}, p\rangle=\omega^{2}|\mathbf{k}, p\rangle .
$$

In the above expression, $\underline{\epsilon}_{0}$ and $\delta \underline{\epsilon}$ are the homogeneous and inhomogeneous part of the dielectric tensor $\underline{\epsilon}$. As can be shown, the operator $\underline{\epsilon}^{-1} \nabla \times \nabla \times$ is Hermitian with respect to the tensor-weighted inner product [Eq. (2)], so that the corresponding eigenvectors $|\mathbf{k}, p\rangle$ are orthogonal, and the $\omega^{2}(\mathbf{k}, p)$ are real.

In the case of $\delta \underline{\epsilon}=0$ we obtain the eigenvalue $\omega_{0}(\mathbf{k}, p)$, describing the dispersion relation for the propagation of linearly polarized $\sigma$ and $\pi$ waves in a birefringent, uniaxial medium. These eigenwaves will be denoted as $|\mathbf{k}, \sigma\rangle$ and $|\mathbf{k}, \pi\rangle$, respectively. The presence of a small periodic perturbation in Eq. (3) significantly alters the situation. In particular, one has to introduce the concept of a Brillouin zone whereas the eigensolutions should obtain the Bloch form. Moreover, on the basis of general considerations, one can expect that the periodic perturbation would have the strongest influence at the degeneration points $\omega_{0}(\mathbf{k}, \sigma)=\omega_{0}(\mathbf{k}, \pi)$, which is consistent with the analysis of Oldano, Allia, and Trossi [8].

After applying a standard perturbative approach to Eq. (3), one obtains a first-order corrected eigenvalue $\omega(\mathbf{k}, p)$, 


$$
\omega^{2}(\mathbf{k}, p)=\omega_{0}^{2}(\mathbf{k}, p)\left[1-\frac{\left\langle\mathbf{k}, p\left|\underline{\epsilon}_{0}^{-1} \delta \underline{\epsilon}\right| \mathbf{k}, p\right\rangle}{\langle\mathbf{k}, p \mid \mathbf{k}, p\rangle}\right] \quad p=\sigma, \pi .
$$

In the above expression, the second term in brackets is just the space-averaged value of the inhomogeneous part of the dielectric tensor $\delta \underline{\epsilon}(z)$. Higher-order corrections to the eigenvalue $\omega(\mathbf{k}, p)$ include terms proportional to

$$
\left|\left\langle\mathbf{k}^{\prime}, p^{\prime}\left|\underline{\epsilon}_{0}^{-1} \delta \underline{\epsilon}\right| \mathbf{k}, p\right\rangle\right|^{2} \propto \theta^{4}
$$

and are important near the points of degeneration, i.e., near the edges of the Brillouin zone, introduced by the periodic form of the perturbation $\delta \underline{\epsilon}(\mathbf{r})$.

The above expression for the first-order corrected eigenvalue $\omega^{2}(\mathbf{k}, p)$ in the presence of the perturbation $\delta \underline{\epsilon}(z)$ can be interpreted as a change in the refractive indices of the medium. This means that in the first-order approximation and far from the degeneration points, the optical properties are described by the corresponding space-averaged uniaxial tensor $\langle\underline{\epsilon}\rangle$. As can be deduced from Eqs. (1) and (4), the first-order correction preserves the form of the normal wave surface, whereas the diagonal elements of the corresponding space-averaged tensor $\langle\underline{\epsilon}\rangle$ are

$$
\begin{aligned}
\langle\underline{\epsilon}\rangle_{x x}= & \frac{1}{2}\left(\epsilon_{1}+\epsilon_{2}\right)+\frac{1}{2}\left(\epsilon_{3}-\epsilon_{1}\right) \theta^{2} \\
& +\frac{1}{2}\left[\left(\epsilon_{1}-\epsilon_{2}\right)+\left(\epsilon_{3}-\epsilon_{1}\right) \theta^{2}\right]\langle\cos 2 \Phi(z)\rangle, \\
\langle\underline{\epsilon}\rangle_{y y}= & \frac{1}{2}\left(\epsilon_{1}+\epsilon_{2}\right)+\frac{1}{2}\left(\epsilon_{3}-\epsilon_{1}\right) \theta^{2} \\
& -\frac{1}{2}\left[\left(\epsilon_{1}-\epsilon_{2}\right)+\left(\epsilon_{3}-\epsilon_{1}\right) \theta^{2}\right]\langle\cos 2 \Phi(z)\rangle, \\
\langle\underline{\epsilon}\rangle_{z z}= & \epsilon_{3}-\left(\epsilon_{3}-\epsilon_{1}\right) \theta^{2} .
\end{aligned}
$$

Note that in the case of the undistorted helicoidal phase, where $\Phi(z)=(2 \pi / p) z,\langle\cos 2 \Phi\rangle=0$, the dielectric tensor $\langle\epsilon\rangle$ [Eqs. (5)-(7)] reduces to the uniaxial tensor with the optical axis parallel to the helical axis. A similar firstorder approximation, although not explicitly derived from the wave equation, was used for the description of the conoscopic figure of the $\mathrm{Sm}-\mathrm{C}^{*}$ phase of a ferroelectric liquid crystal $[11,12]$.

We shall consider now the effect of an external magnetic field, perpendicular to the helical axis on the optical properties of the $\mathrm{Sm}-C_{A}^{*}$ phase. This analysis is similar to the case of a ferroelectric Sm- $C^{*}$ phase, due to the same diamagnetic coupling. As it is well known from the theory of the ferroelectric Sm- $C^{*}$ phase in a transverse magnetic field $[13,14]$, the phase profile $\Phi(z)$ of the distorted helicoidal structure obtains a $\pi$-soliton form $\sin \Phi(z)=\operatorname{sn}(u, k)$, where $\operatorname{sn}(u, k)$ is the Jacobian sine amplitude with the argument $u=z /(\xi k)$ and the coherence length $\xi^{2}=K_{33} /\left(\Delta \chi H^{2}\right)$. Here $K_{33}$ is the torsional elastic constant, $\Delta \chi>0$ is the diamagnetic anisotropy, and the direction of external field is $\mathbf{H}=(0, H, 0)$. Modulus $k$ of the Jacobian sine amplitude is defined by $k=\left(H / H_{c}\right) E(k)$, where $H_{c}=\left(\pi^{2} / p_{0}\right) \sqrt{K_{33} / \Delta \chi}$ is the critical field for the unwinding of the helical structure and $E(k)$ is the complete elliptic integral of the second kind.
The components $\langle\underline{\epsilon}\rangle_{x x}$ and $\langle\underline{\epsilon}\rangle_{y y}$ of the spaceaveraged dielectric tensor of the antiferroelectric phase depend on the magnitude of the external magnetic field $H$ through the space-averaged values of $\langle\cos 2 \Phi(z)\rangle$ $=1-2\left\langle\operatorname{sn}^{2}(u, k)\right\rangle$. More precisely, the space-averaged value of the dielectric tensor of the Sm- $C_{A}^{*}$ phase in an external field is given by

$$
\langle\underline{\epsilon}(H)\rangle=\left[\begin{array}{ccc}
\epsilon_{x}-\Delta(H) & 0 & 0 \\
0 & \epsilon_{x}+\Delta(H) & 0 \\
0 & 0 & \epsilon_{z z}
\end{array}\right] .
$$

Here, $\epsilon_{x}=\frac{1}{2}\left(\epsilon_{1}+\epsilon_{2}\right)+\frac{1}{2}\left(\epsilon_{3}-\epsilon_{1}\right) \theta^{2}$ is the magnetic-field independent part, whereas the magnetic-field dependent part is

$\Delta(H)=\frac{1}{2}\left[\left(\epsilon_{1}-\epsilon_{2}\right)+\left(\epsilon_{3}-\epsilon_{1}\right) \theta^{2}\right]\left[2 \frac{K(k)-E(k)}{k^{2} K(k)}-1\right]$.

In the above expression, $K(k)$ is the complete elliptic integral of the first kind. In the limit of small field, $H \rightarrow 0$, $\Delta(H) \rightarrow 0$, whereas near the critical field $H \rightarrow H_{c}$, $\Delta(H) \rightarrow \frac{1}{2}\left[\left(\epsilon_{1}-\epsilon_{2}\right)+\left(\epsilon_{3}-\epsilon_{1}\right) \theta^{2}\right]$. As a consequence, in the limit of zero field, $\langle\underline{\epsilon}\rangle$ [Eq. (8)] obtains a uniaxial form, whereas in the presence of a transverse magnetic field $\langle\underline{\epsilon}\rangle$ obtains a biaxial form. This is an example of magnetic-field induced biaxiality in a distorted helicoidal phase. It should be stressed that the magnetic-fieldinduced biaxiality is here mainly the result of the global distortion of the phase of the order parameter and not the result of the intrinsic local biaxiality of the liquid-crystal molecules.

The above model [Eq. (8)] predicts an apparent reduction of the principal value $\langle\underline{\epsilon}\rangle_{x x}$, whereas $\langle\underline{\epsilon}\rangle_{y y}$ is expected to increase with increasing magnetic field. Although the overall changes of the components of $\langle\underline{\epsilon}\rangle$ are expected to be small, they should be observable at fields far below the critical field. In particular, magnetic-fieldinduced biaxiality in an antiferroelectric phase with $\Delta \chi>0$ should be observable as a magnetic-field-induced distortion of the conoscopic figure in a plane, perpendicular to the direction of the magnetic field, i.e., the $x-z$ plane. Figures 1(a) and 1(b) show the influence of an external magnetic field on the $x-z$ cross sections of the normal wave surface of an antiferroelectric liquidcrystalline phase. In zero field [Fig. 1(a)], the section of a normal wave surface at $y=0$ consists of a circle and an oval, corresponding to the unperturbed ordinary $(\sigma)$ and unperturbed extraordinary $(\pi)$ wave, with the optical axis along $z$. The magnetic-field dependence of the ordinary and extraordinary indices of refraction for the propagation of light in the $x-z$ plane are deduced from Eqs. (8) and (9) as

$$
\begin{aligned}
& n_{o}^{2}=\langle\epsilon\rangle_{y y}(H)=\epsilon_{x}+\Delta(H), \\
& \frac{1}{n_{e}^{2}}=\frac{\sin ^{2} \alpha}{\langle\epsilon\rangle_{z z}}+\frac{\cos ^{2} \alpha}{\epsilon_{x}-\Delta(H)} .
\end{aligned}
$$

Here, $\alpha$ is the angle between the direction of wave propa- 


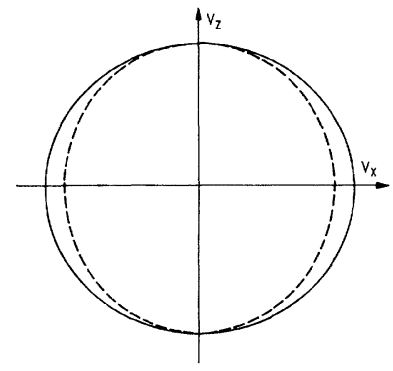

(a) $\vec{H}=0$

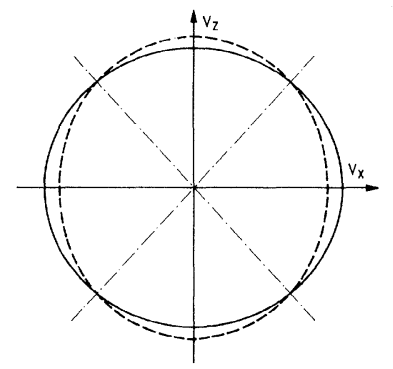

(b) $\vec{H}=(0, H, O) \neq 0$
FIG. 1. (a) $x-z$ cross section of the normal surface of an antiferroelectric liquid crystal in first-order approximation at $H=0$ and (b) magnetic-field-induced distortion of the $x-z$ cross section of the normal surface in the first-order approximation. Note the splitting of a single optical axis into two optical axes.

gation and the $z$ axis. According to Eq. (10), under the influence of an external field, the circle, corresponding to the phase velocity of the ordinary wave $c=c_{o} / n_{o}$, contracts with increasing magnetic field, whereas the oval [Eq. (11)] is deformed in the $z$ direction, as is shown in Fig. 1(b). The single optical axis at $H=0$ splits into two optical axes, located symmetrically with respect to the $z$ axis. As a result of this splitting, the conoscopic figure, which reflects the shape of the two normal wave surfaces, is strongly deformed in the $x-z$ plane. The deformation is most pronounced near the center of the conoscopic figure, where even a small change in the index of refraction induces a strong splitting between the two optical axes. Finally, very near the critical field, the angle $\beta$ between the two optical axis should saturate near the value $\operatorname{tg}(\beta / 2) \approx \sqrt{\epsilon_{3} / \epsilon_{1}} \theta$.

The position $\alpha_{n}$ of the $n$th interference minimum of the $x-z$ cross section of the conoscopic figure is deduced from Eqs. (10) and (11) and satisfies the equation

$$
\begin{array}{r}
\frac{\sqrt{\langle\epsilon\rangle_{x x}(H)}}{\left[1-\left[1-\frac{\langle\epsilon\rangle_{x x}}{\langle\epsilon\rangle_{z z}}\right] \sin ^{2} \alpha_{n}\right]^{1 / 2}}-\sqrt{\langle\epsilon\rangle_{y y}(H)} \\
-n \frac{\lambda_{0}}{d_{0}}\left(1-\sin ^{2} \alpha_{n}\right)^{1 / 2}=0 .
\end{array}
$$

Here, $\lambda_{0}$ is the vacuum wavelength of the incident light, $d_{0}$ is the thickness of the sample, $\langle\underline{\epsilon}\rangle_{i i}, i=x, y, z$ are defined in Eqs. (8) and (9), and the sample is placed between crossed polarizers.

\section{EXPERIMENT}

In the experiment we have used a scanning-conoscopic technique to measure the intensity distribution in a plane, perpendicular to the magnetic field. The conoscopic figure was measured by recording the angular dependence of the intensity of light, transmitted through the homeotropic Sm- $C_{A}^{*}$ sample, placed between crossed polarizers with the axis of polarization at $45^{\circ}$ with respect to the field direction and the plane of incidence.

The experiment was performed in a miniaturized optical setup (Fig. 2), fitting into a $60-\mathrm{mm}$ bore of a Bitter magnet. The beam of a 10-mW He-Ne laser was expanded to $10-\mathrm{mm}$ diameter and then slightly focused onto the sample, placed in the center of the magnet. A system of mirrors was used for fine adjustment of the optical path in the setup. We avoided the use of glass prisms because of the relatively high values of Faraday rotation of the polarization of light in glass at high magnetic fields. The light, transmitted through the sample placed between crossed polarizers, was collected with a lens and focused into a multimode fiber. The intensity was measured by a photodiode and recorded on an $x-y$ recorder.

The sample cell was prepared by treating clean glass plates with dimethyloctadecyl-3-(trimethoxysilyl)propylammonium chloride (DMOAP). Good homeotropic

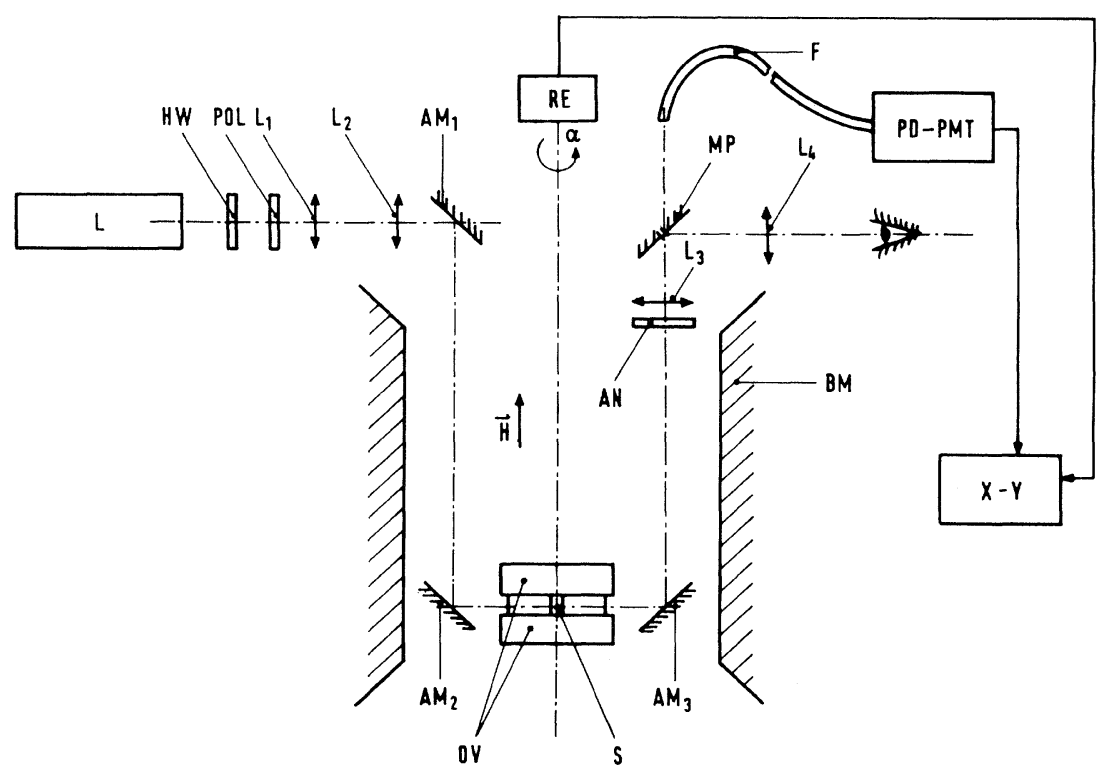

FIG. 2. Optical setup for scanning the conoscopic figure of an antiferroelectric liquid crystal in a transverse magnetic field. Definitions: L, laser; HW, half-wave plate; POL, polarizer; $L_{1}, L_{2}, L_{3}, L_{4}$, lenses; $\mathrm{AM}_{1}, \mathrm{AM}_{2}, \mathrm{AM}_{3}$, adjustable mirrors; $\mathrm{OV}$, oven; S, sample; AN, analyzer; MP, mirror with a pinhole; F, fiber; PD-PMT, photodiodephotomultiplier; RE, rotary encoder; $X-Y, x-y$ recorder; BM, Bitter magnet. 
alignment was obtained in $50-\mu \mathrm{m}$-thick cells, filled with 4-(1-methyl-heptyloxycarbonylphenyl) 4'-octylbiphenyl-4-carboxylate (MHPOBC). The sample was placed between two glass half-cylinders, thus forming a cylinder with the axis parallel to the direction of the magnetic field and perpendicular to the helical axis of the Sm- $C_{A}^{*}$ phase. An index-matching resin was used to minimize the reflections from the air-glass interfaces. The glass cylinder was put in a temperature-stabilized oven $( \pm 0.1 \mathrm{~K})$, placed in the center of the magnet. The oven and the cylinder could be rotated around the axis of the cylinder, i.e., the $y$ axis. The angle of rotation of the sample $(\alpha)$ was measured by a rotary encoder that was connected to the $x-y$ recorder. The construction of the setup assured the condition $\mathbf{q}_{c} \perp \mathbf{H}$ at all angles of the rotation of the sample. Here $\mathrm{q}_{c}$ is the wave vector of the $\mathrm{Sm}-C_{A}^{*}$ phase. It should be noted that the direction of light propagation through the glass cylinder in the center of the magnet was perpendicular to the direction of the magnetic field, so that Faraday rotation of the light polarization in glass was avoided.

\section{RESULTS AND DISCUSSION}

Figure 3 shows the magnetic-field dependence of the conoscopic figure of MHPOBC in the Sm- $C_{A}^{*}$ phase. At

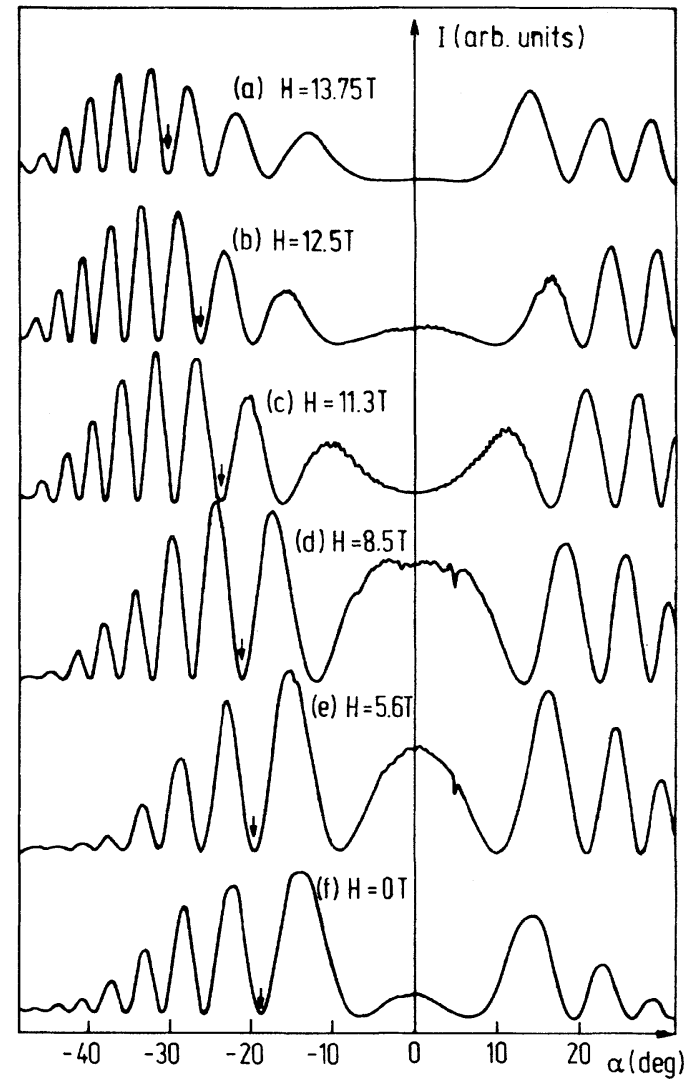

FIG. 3. Magnetic-field dependence of the intensity distribution of the $x-z$ cross section of the conoscopic figure in the Sm$C_{A}^{*}$ phase of MHPOBC at different magnetic fields. The temperature is $89^{\circ} \mathrm{C}$. The arrow indicates the position of $n=1$ fringe. zero magnetic field the transmitted light intensity distribution [Fig. 3(f)] is typical for an optically uniaxial medium with some optical rotation $[1,12]$ of the polarization of light, propagating at small angles with respect to the optical axis. This is reflected in an increased transmission at normal incidence, resulting in a "bright" central region of the conoscopic figure. The shape of the conoscopic figure of the Sm- $C_{A}^{*}$ phase changes significantly already at intermediate values of the magnetic field. As is shown in Fig. 3, the bright central part of the conoscopic figure becomes broader, shifting the neighboring minima of the interference pattern towards larger values of the angle of incidence. At the same time, the distortion of the conoscopic figure is significant near normal incidence, whereas at higher angles of incidence it remains practically unchanged. Very near the critical field for the unwinding of the helical structure, but still in the helical phase, the distorted central part of the conoscopic figure has almost doubled in size. Above the critical field $H_{c}$, the conoscopic figure is very similar to the undistorted Sm- $C_{A}^{*}$ one and changes only slightly upon increasing magnetic field. A similar behavior was observed by Gorecka et al. [2] and others [1,3], when studying electric-field effects of the Sm- $C_{A}^{*}$ phase of MHPOBC.

The magnetic-field dependence of the angular position of the first three light intensity minima, as measured at two different temperatures in the $\mathrm{Sm}-C_{A}^{*}$ phase of MHPOBC, are shown in Figs. 4 and 5, respectively. One immediately observes that the magnetic-field-induced shift of these minima is indeed very large at low interference orders, corresponding to small zero-field retardations of both interfering eigenwaves. At larger values of zero-field retardation, corresponding to large incidence angles ( $n=3$, Fig. 4), the positions of the minima of the light distribution change much less upon increasing magnetic field. In addition, by comparing the data on Figs. 4 and 5 , one can observe a very strong temperature dependence of the critical magnetic field $H_{c}$, necessary to unwind the helical structure.

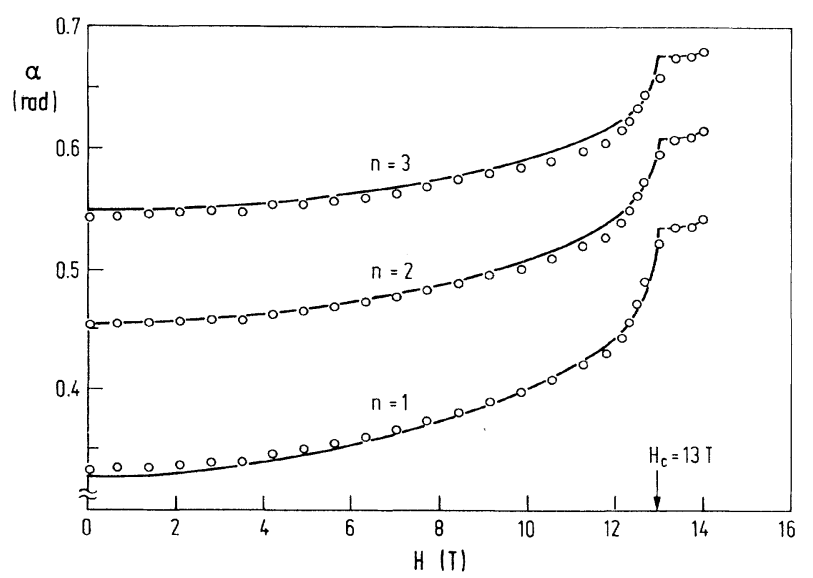

FIG. 4. Magnetic-field dependence of the first three intensity minima of the $x-z$ cross section of the conoscopic figure at $T=89^{\circ} \mathrm{C}$. The solid line is the best fit to Eq. (12) with $H_{c}=13 \pm 0.3 \mathrm{~T}$ and $\delta \Delta=0.046 \pm 0.002$. 


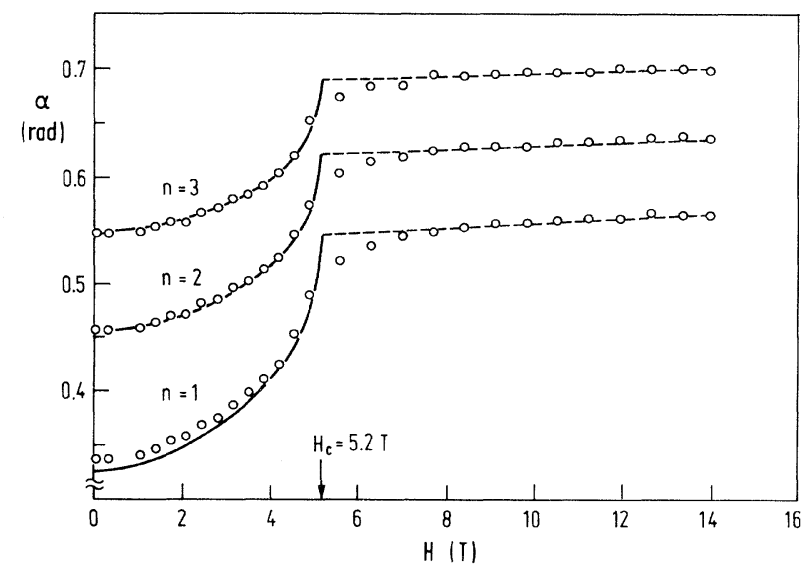

FIG. 5. Magnetic-field dependence of the first three intensity minima of the $x-z$ cross section of the conoscopic figure at $T=80^{\circ} \mathrm{C}$. The solid line is the best fit to Eq. (12) with $H_{\mathrm{c}}=5.2 \pm 0.2 \mathrm{~T}$ and $\delta \Delta=0.052 \pm 0.002$.

The data of Figs. 4 and 5 were fitted to Eq. (12), taking

$$
\delta \Delta=\Delta\left(H_{c}\right)-\Delta(0)=\frac{1}{2}\left[\left(\epsilon_{1}-\epsilon_{2}\right)+\left(\epsilon_{3}-\epsilon_{1}\right) \theta^{2}\right]
$$

and the critical field $H_{c}$ as the fitting parameters. The zero-field optical anisotropy was chosen as $\Delta n=n_{e}-n_{o}=0.12$ [2]. As one can see from the solid lines in Fig. 4, the agreement between the theoretically predicted magnetic-induced shift (solid lines) and the measured shift of the light-intensity minima is excellent. The fitting procedure yields $H_{c}=13 \pm 0.3 \mathrm{~T}$ and $\delta \Delta=0.046 \pm 0.002$ at $T=89^{\circ} \mathrm{C}$ (Fig. 4), whereas at $80^{\circ} \mathrm{C}$ we obtain $H_{c}=5.2 \pm 0.2 \mathrm{~T}$ and $\delta \Delta=0.052 \pm 0.002$ (Fig. 5). Choosing [2] $\epsilon_{1}=2.25, \sqrt{\epsilon_{2}}-\sqrt{\epsilon_{1}}=3 \times 10^{-3}, \theta=24^{\circ}$ and $\Delta n=0.12$, and taking into account $\theta^{2}$ dependence [see Eq. (1)] of the extraordinary index of refraction, we calculate from Eq. (1) $\epsilon_{3}=2.74$ and $\delta \Delta=0.038$ at $T=89^{\circ} \mathrm{C}$, which is close to the experimentally observed value $\delta \Delta=0.046$. By lowering the temperature, $\delta \Delta$ increases slightly. This is consistent with the increase of the tilt angle $\theta$, which gives the main contribution to the biaxiality parameter $\delta \Delta$.

The measurements of the magnetic-field-induced biaxiality also allow for the determination of the critical field for the unwinding of the helical structure. The period of the double-helical structure of MHPOBC has been determined by selective reflection spectra [1]. It is equal to 1.5 $\mu \mathrm{m}$ at $89^{\circ} \mathrm{C}$ and seems to diverge at $80^{\circ} \mathrm{C}$. The product $H_{c} p$, which determines the temperature dependence [13] of the critical field $H_{c}$ is equal to $H_{c} p=19.5 \pm 0.5 \mathrm{~T} \mu \mathrm{m}$ and is comparable to the value $25 \mathrm{~T} \mu \mathrm{m}$, as obtained for DOBAMBC [13]. As the period of the Sm- $C_{A}^{*}$ phase of MHOPBC shows a strong temperature dependence [1], we expect a strong temperature dependence of the critical magnetic field. This was indeed observed in the experiment. At $T=80^{\circ} \mathrm{C}$ (Fig. 5) the critical field is as low as 5.2 $\mathrm{T}$, whereas at $T=94^{\circ} \mathrm{C}$ the critical magnetic field is beyond the highest accessible value of $14 \mathrm{~T}$ in the present experiment.

The magnetic-field dependence of the conoscopic figure can also be well explained with a simple and intuitive consideration of what happens with the helical structure and the corresponding dielectric tensor, when the transverse magnetic field is switched on. In the case of positive diamagnetic anisotropy, the field tends to align the molecules in the direction of the field. This results in a molecular distribution, where most of the molecules are tilted in a plane, parallel to the field and to the layer normal. As a result, the space-averaged dielectric constant $\left\langle\epsilon_{y y}(H)\right\rangle$ increases with increasing field whereas the average dielectric constant $\left\langle\epsilon_{x x}(H)\right\rangle$ decreases with increasing field, because only few molecules are tilted in a direction, perpendicular to the field. This results in magnetic-field-induced biaxiality, where the two optical axis of the biaxial structure appear [15] in the $x-z$ plane, i.e., in the plane, perpendicular to the magnetic-field direction. The half value of the angle between the two optical axis is field dependent and can be shown [15] to saturate near the value of the tilt angle $\theta$ as one approaches the critical field $H_{c}$. This explains the strong shift of the intensity minima near the center of the conoscopic figure. It is thus a result of the splitting of a single optic axis of the unperturbed $\mathrm{Sm}-C_{A}^{*}$ phase into two optical axes of the deformed Sm- $C_{A}^{*}$ phase, which are separated by a field-dependent angle.

\section{CONCLUSION}

The distortion of the conoscopic figure of the $\mathrm{Sm}-C_{A}^{*}$ phase in an external magnetic field can be well understood within the framework of a first-order perturbative approximation of the dispersion relation $\omega(\mathbf{k})$ for the optical eigenmodes. The observed magnetic-field-induced biaxiality of the $\mathrm{Sm}-C_{A}^{*}$ reflects the appearance of the solitonlike distortion of the phase of the order parameter, induced by the external magnetic field. It should be stressed that the electric-field-induced distortion of the conoscopic figure, which has been observed in the Sm- $C_{A}^{*}$ of MHPOBC [2], and the distortion of the conoscopic figure by the linear PE coupling should have the same physical origin and could be similarly explained by the appearance of phase solitons.

\section{ACKNOWLEDGMENT}

The authors would like to thank Professor Y. Ishibashi for the MHPOBC sample.
[1] A. D. L. Chandani, E. Gorecka, Y. Ouchi, H. Takezoe, and A. Fukuda, Jpn. J. Appl. Phys. 28, L1265 (1989).

[2] E. Gorecka, A. D. L. Chandani, Y. Ouchi, H. Takezoe, and A. Fukuda, Jpn. J. Appl. Phys. 29, 131 (1990).

[3] K. Hiraoka, A. D. L. Chandani, E. Gorecka, Y. Ouchi, H.
Takezoe, and A. Fukuda, Jpn. J. Appl. Phys. 29, L1473 (1990).

[4] S. Garoff, R. B. Meyer, and R. Barakat, J. Opt. Soc. Am. 68, 1217 (1978).

[5] D. W. Berreman, Mol. Cryst. Liq. Cryst. 22, 175 (1973). 
[6] P. G. de Gennes, The Physics of Liquid Crystals (Clarendon, Oxford, 1974).

[7] C. Oldano, Phys. Rev. Lett. 53, 2413 (1984).

[8] C. Oldano, P. Allia, and L. Trossi, J. Phys. (Paris) 46, 573 (1985).

[9] I. Abdulhalim and L. Benguigui, Ferroelectrics 84, 273 (1988).

[10] M. A. Peterson, Phys. Rev. A 27, 520 (1983).

[11] Pawel Pierański, E. Guyon, P. Keller, L. Liébert, W. Kuczynski, and Piotr Pieranski, Mol. Cryst. Liq. Cryst. 38,
275 (1977).

[12] D. Taupin, E. Guyon, and P. Pieranski, J. Phys. (Paris) 39, 406 (1978).

[13] R. Blinc, I. Muševič, and B. Žekš, Phys. Scr. T35, 38 (1991).

[14] I. Muševič, B. Žekš, R. Blinc, H. A. Wierenga, and Th Rasing, Phys. Rev. Lett. 68, 1850 (1992).

[15] M. Born and E. Wolf, Principles of Optics (Pergamon, New York, 1959). 